\title{
FREQUENCY DOUBLER AND TWO-COLOR MODE OF OPERATION AT FREE ELECTRON LASER FLASH2
}

\author{
M. Kuhlmann, E.A. Schneidmiller and M.V. Yurkov, DESY, Hamburg, Germany
}

\begin{abstract}
We report on the results of the first operation of a frequency doubler at FLASH2. The scheme uses the feature of the variable gap of the undulator. Undulator is divided in two parts. The second part of the undulator is tuned to the double frequency of the first part. Modulated electron beam enters the second part of the undulator and generates radiation at the 2 nd harmonic. Depending on a balance between undulator sections, frequency doubler allows operation in a two-color mode and operation at shorter wavelengths with respect to standard SASE scheme. The shortest wavelength of $3.1 \mathrm{~nm}$ (photon energy $400 \mathrm{eV}$ ) has been achieved at FLASH2 with frequency doubler scheme, which is significantly below the design value for the standard SASE option.
\end{abstract}

\section{INTRODUCTION}

Free electron laser FLASH is equipped with two undulator beamlines [1]. Fixed gap undulator (period $2.73 \mathrm{~cm}$, peak magnetic field $0.48 \mathrm{~T}$, total magnetic length $27 \mathrm{~m}$ ) is installed in the first beamline, FLASH1. The second beam line, FLASH2, is equipped with variable gap undulator (period $3.14 \mathrm{~cm}$, maximum peak magnetic field $0.96 \mathrm{~T}$, total magnetic length $30 \mathrm{~m}$ ). With operating range of the electron beam energies of $0.4-1.25 \mathrm{GeV}$ FLASH1 and FLASH 2 beamline cover wavelength range from 4-52 $\mathrm{nm}$ and 3.5$90 \mathrm{~nm}$, respectively. Scope of scientific applications will benefit a lot with extension of the operating range of the facility deep into the water window spanning from $4.38 \mathrm{~nm}$ and $2.34 \mathrm{~nm}$ (K-absorption edges of carbon and oxygen).

One of the techniques to generate shorter wavelength is the second harmonic afterburner [2-7]. Afterburner operating at the 2 nd harmonic has been tested successfully at LCLS with 5 final undulator modules retuned to the 2nd harmonic [7]. With long, variable gap undulator of FLASH2 it is possible to implement frequency doubler scheme. Here we demonstrate experimental results from FLASH2: with an appropriate optimization of undulator tuning it becomes possible to operate facility at visibly shorter wavelengths and organize two color mode of operation $(\omega+2 \omega)$ with controllable radiation pulse intensities.

\section{OPERATION OF FREQUENCY DOUBLER}

Undulator is divided undulator in two sections tuned to $\omega$ and $2 \omega$ frequency. The plots in Figure 1 illustrate general features of the operation of the frequency doubling scheme. Black dashed line shows evolution of the radiation power along the $\omega$-section. Amplification process stops at some length, and then electron beam enters the $2 \omega$-section tuned to the doubled frequency. Radiation with frequency $\omega$ does not interact with the electron beam in the $2 \omega$-section, and just propagates forward. Radiation power produced in the $\omega$-section at the second harmonic frequency is significantly suppressed $[6,8]$, thus only the beam density modulations at $2 \omega$ frequency can seed the $2 \omega$-section.

We performed simulations of the amplification process for the full parameter space of the lengths of the $\omega$ and $2 \omega$ sections, and results are summarized in Fig. 2. Despite simulations have been performed for specific set of electron beam parameters, presentation of the results in normalized form allows to use them in a wider range of parameters. Xcoordinate is radiation power from the $\omega$-section normalized to the saturation power of SASE FEL operating at frequency $\omega$. Y-coordinate is radiation power from the $2 \omega$ section normalized to the saturation power of SASE FEL operating at frequency $2 \omega$. We see that with two-color mode of operation it is possible to produce simultaneously high power at both frequencies with the level of about $20 \%$ of the saturation power. When amplification process in the first undulator terminates at the onset of the nonlinear regime, it is possible to generate high radiation power at the second harmonic in the second part of the undulator.

Simple physical considerations explain operation of the frequency doubler. Indeed, for a short length of the $\omega$ section, when amplification process stays in the exponential gain regime, the beam density modulations at frequency $2 \omega$ remain on the level of the shot noise. Induced modulations

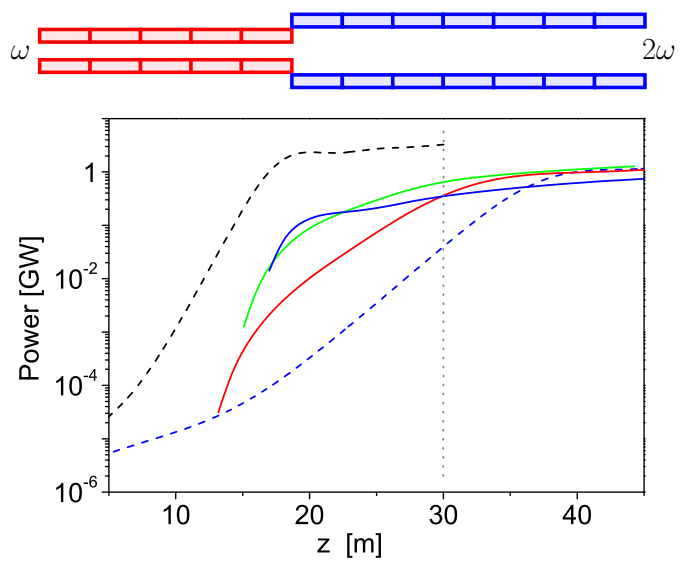

Figure 1: Top: Conceptual scheme of frequency doubler with variable gap undulator. Bottom: Evolution of the radiation power along FLASH2 undulator. Dashed curves correspond to SASE FEL operating at $8 \mathrm{~nm}$ (black) and at $4 \mathrm{~nm}$ (blue). Solid curves correspond to frequency doubler $8 \mathrm{~nm} \rightarrow 4 \mathrm{~nm}$ for different lengths of the $\omega$ undulator section (red, green, and blue colors). Gray dashed line at $z=30 \mathrm{~m}$ shows magnetic length of FLASH2 undulator. Electron energy is $1080 \mathrm{MeV}$, beam current is $1500 \mathrm{~A}$, normalized rms emittance $1 \pi \mathrm{mm}$-mrad, and rms energy spread 0.15 $\mathrm{MeV}$. Simulations have been performed with code FAST [9]. 


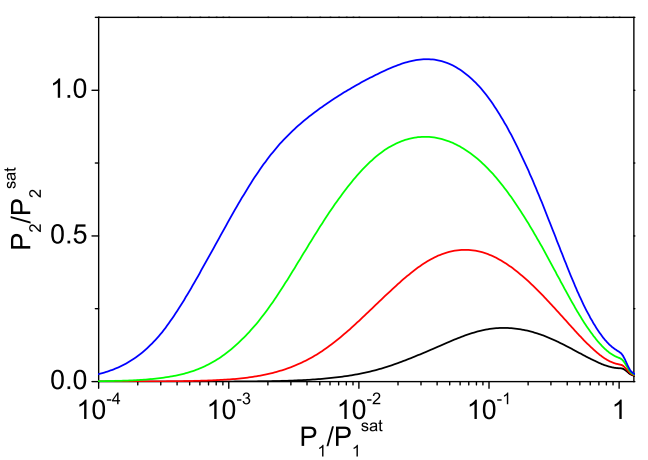

Figure 2: Parameter space of radiation powers from frequency doubler at FLASH2. $P_{1}$ is radiation power at $\omega$ at the exit of the first section, and $P_{2}$ is radiation power at $2 \omega$ at the exit of the doubling section. Radiation powers are normalized to the corresponding saturation powers of SASE FEL. Different colors (black, red, green, and blue) denote lengths of the doubling section in terms of the saturation length: $1 / 6 z_{\mathrm{sat}}, 1 / 3 z_{\mathrm{sat}}, 1 / 2 z_{\mathrm{sat}}$, and $2 / 3 z_{\mathrm{sat}}$. Simulations have been performed with code FAST [9]

at the frequency $2 \omega$ (and higher harmonics) occur only in the nonlinear stage of amplification. At the onset of nonlinear regime induced modulation at $2 \omega$ is small, and gradually grows when amplification process approaches saturation. At the same time we occur degradation of the electron beam quality due to interaction of the electron beam with radiation. Thus, we deal with two competing effects: growth of the beam modulation, and degradation of the electron beam quality. Lucky factor for the frequency doubling scheme is that the growth of the beam density modulation advances the degradation of the beam quality, since the radiation power should be produced first, and only then it interacts with the beam. When amplification process enters the post-saturation regime, the quality of the beam and the beam bunching degrade such that the electron beam becomes to be not appropriate for efficient radiation of higher harmonics.

\section{EXPERIMENTAL RESULTS}

Operation of frequency doubler has been successfully demonstrated during several test runs at FLASH2 in 2016. Experiments have been performed at the energy of the electron beam of $1080 \mathrm{MeV}$ and $1230 \mathrm{MeV}$. Tuning of the frequency doubler scheme with variable gap undulator does not require any special techniques. First, we tune maximum SASE gain in the uniform undulator tuned to frequency $\omega$. Analysis of the gain curve and fluctuations of the radiation pulse energy allows to determine optimum length of the $\omega$ section. Then we tune remaining sections to the frequency $2 \omega$, and after adjustment of the phase shifters and electron beam orbit, the frequency doubler starts to generate radiation at the second harmonic.

Figure 3 shows the gain curve of the frequency doubler $8 \mathrm{~nm} \rightarrow 4 \mathrm{~nm}$. First five undulator sections were tuned to $8 \mathrm{~nm}$, and the last seven sections were tuned to $4 \mathrm{~nm}$. Red and blue curves show radiation pulse energies at $8 \mathrm{~nm}$
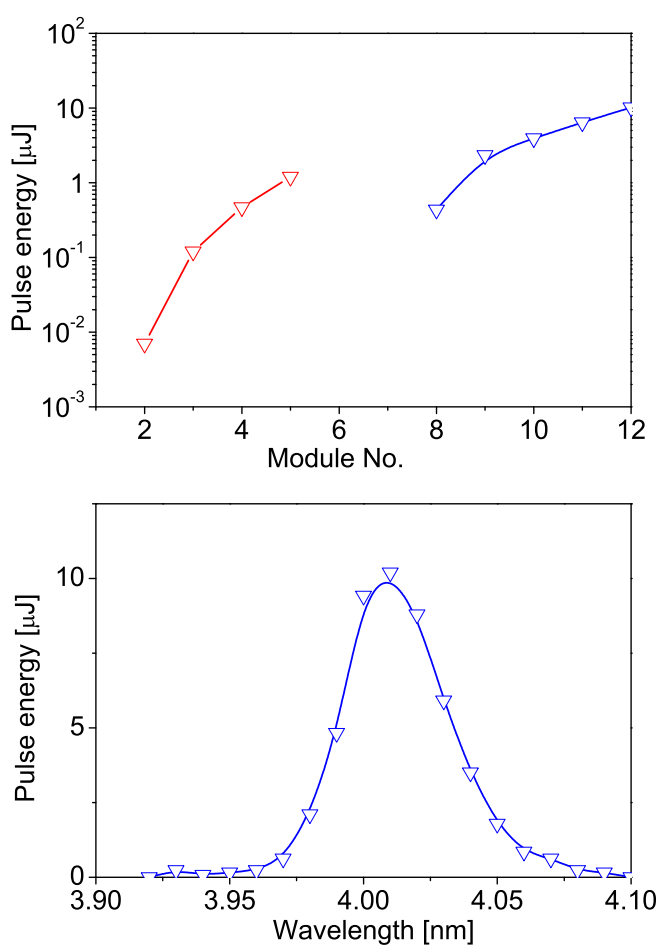

Figure 3: Frequency doubler at FLASH2 (experimental data). Left: gain curve of the frequency doubler at FLASH 2. First part of the undulator ( 5 modules) is tuned to $8 \mathrm{~nm}$, and second part (7 modules) is tuned to $4 \mathrm{~nm}$. Red and blue color correspond to the radiation wavelength of $8 \mathrm{~nm}$ and $4 \mathrm{~nm}$, respectively. Right: radiation pulse energy at the 2 nd harmonic versus resonance frequency of the second part of the undulator. Electron energy is $1080 \mathrm{MeV}$, bunch charge is $300 \mathrm{pC}$.

and $4 \mathrm{~nm}$, respectively. Amplification process in the second part (frequency doubler) exhibits resonance behavior on the frequency detuning (right plot in Fig. 3). Even harmonics of the radiation are strongly suppressed in the planar undulator, thus we get confirmation that the beam bunching at the second harmonic seeds the amplification process of the frequency doubling sections. One can compare experimental and simulation results presented in Fig. 1. Gain curves look pretty much similar. As for the resonance curve, the width of experimental curve is significantly wider than FEL parameter $\rho$. This is typical signature of the energy chirp along the electron beam induced by the beam formation system.

Our experience shows that tuning procedure of the frequency doubler is simple and reproducible. In particular, it is possible to tune relative intensities of two colors $(\omega$, $2 \omega$ ) in a wide limits. When tuned to equal intensities, pulse energies were in the range from a few to 10 microjoules. Radiation has been transported to FLASH2 experimental hall for characterization. Figure 4 shows an example for photon beam images and spectra of two color mode of operation for the frequency doubler $9 \mathrm{~nm} \rightarrow 4.5 \mathrm{~nm}$. With this first experience we can state that this operational mode can be proposed to users. 

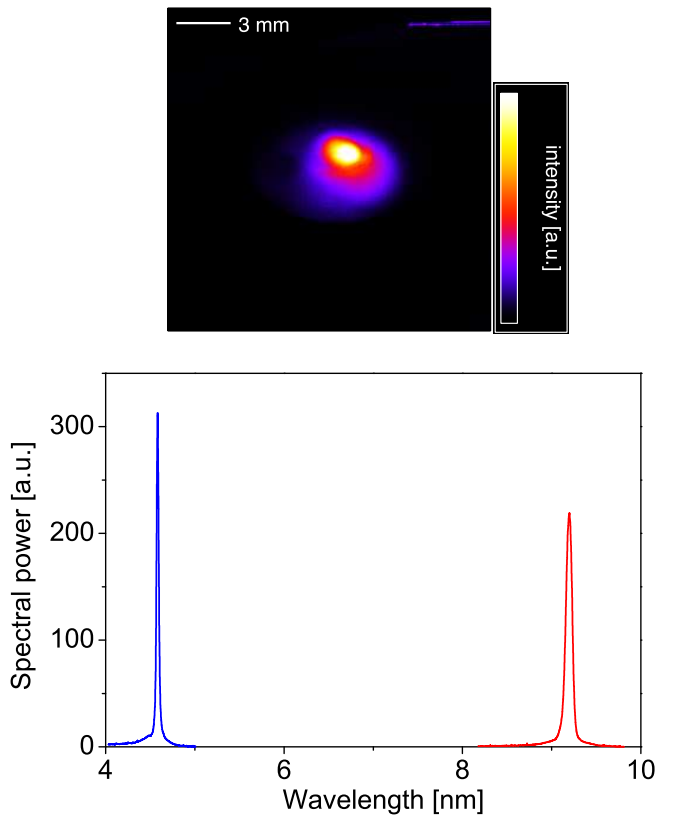

Figure 4: Photon beam image in the experimental hall(top) and radiation spectra (bottom) of the frequency doubler at FLASH2 (experimental data). Small yellow spot is $4.5 \mathrm{~nm}$ (2nd harmonic) radiation, pulse energy is $10 \mathrm{~J}$. Larger blue/pink spot is $9 \mathrm{~nm}$ radiation, pulse energy is $10 \mu \mathrm{J}$. Electron energy is $1080 \mathrm{MeV}$, bunch charge is $300 \mathrm{pC}$.

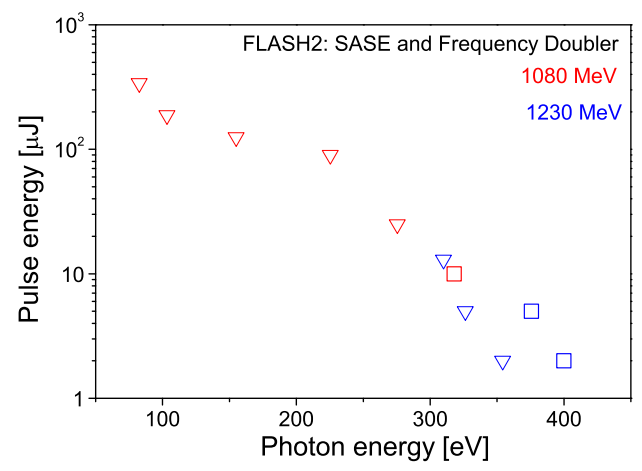

Figure 5: Radiation pulse energy versus photon energy. Experimental data from FLASH2 for electron energy of $1080 \mathrm{MeV}$ and $1230 \mathrm{MeV}$, bunch charge is $300 \mathrm{pC}$. Triangles represent SASE, and squares represent frequency doubler.

With fixed electron energy, shortening of the radiation wavelength is achieved by opening the undulator gap. Undulator field is reduced, the FEL gain falls down, and starting from some wavelength the undulator length is not sufficient to reach saturation regime in the uniform undulator. Frequency doubler scheme is capable to generate shorter wavelength radiation than standard SASE FEL. Indeed, the first part of the undulator operates at twice longer wavelength, and saturation is obtained at a half of the full undulator length. Induced beam bunching at the second harmonic is much larger than the shot noise in the electron beam, and it becomes possible to reach saturation on much shorter length of the doubling section. For fair comparison of two options (SASE and frequency doubler) we performed two dedicated runs at FLASH2 operating with electron energies close to the limit. First, standard SASE mode has been optimally tuned at full undulator length, and radiation pulse energy scanned versus radiation wavelength. Then frequency doubler scheme has been tuned optimally with the same electron beam, and the pulse energy scanned. Results of pulse energy measurements are compiled in Fig. 5. We see that visibly shorter wavelengths were reached with the frequency doubler. In particular, photon energies above Nitrogen K-edge have been demonstrated which significantly exceeds original specifications of FLASH2. The radiation has been successfully transported to FLASH2 experimental hall.

Analysis of experimental results obtained at FLASH2 shows that the frequency doubler scheme works in good agreement with theoretical expectations. Its tuning does not require special efforts, and it starts to work when SASE process tuned along the whole length of the undulator. Two color mode of operation is rather robust for longer wavelengths. Minimum achievable wavelength, strongly depends on the quality of the electron beam which requires dedicated tuning of the beam formation system. However, this is general problem for SASE FEL as well. Presently we reached photon energies just below Nitrogen K-edge (400 $\mathrm{eV}$ ), and this seems to be close to the limit of the doubling frequency technique at he energy of electron beam of 1230 $\mathrm{MeV}$. Additional installation of two undulator sections $(2 \times$ $2.5 \mathrm{~m}$ ) may help in more reliable covering of the Nitrogen K-edge range and reaching shorter wavelengths.

\section{ACKNOWLEDGEMENTS}

We are grateful to B. Faatz, J. Rönsch-Schulenburg, S. Schreiber, M. Tischer for fruitful collaboration, and FLASH team for technical support. We would like to thank R. Brinkmann, J. Schneider, E. Weckert and W. Wurth for valuable discussions and interest in this work.

\section{REFERENCES}

[1] B. Faatz et al., New journal of physics 18(6), 062002 (2016).

[2] R. Bonifacio, L. De Salvo, and P. Pierini, Nucl. Instrum. Methods Phys. Res. A 293, (1990), 627.

[3] Ciocci, et al., IEEE J. Quantum Electron. 31 (1995) 1242.

[4] W. M. Fawley et al., Proceedings of the IEEE 1995 Partice Accelerator Conference, 1996, p. 219.

[5] T. Aberg et al., A VUV Free Electron Laser at DESY: Conceptual Design Report. TESLA FEL Report 1995-03, DESY, Hamburg, 1995.

[6] Z. Huang and S. Reiche, Proc. of the FEL2004 Conference, Trieste, Italy, p. 201, [http://www.jacow.org]

[7] H.-D. Nuhn et al., Proc. of the FEL2010 Conference, Malmo, Sweden, p. 690, [http://www.jacow.org]

[8] G. Geloni et al., Preprint DESY 05-137, Hamburg, 2005.

[9] E.L. Saldin, E.A. Schneidmiller and M.V. Yurkov, Nucl. Instrum. and Methods A 429 (1999) 233. 